\title{
Efficacy of pre-operative cephalosporin prophylaxis in controlling pathogenic oral bacteria growth in comatose patients
}

Saliva contains many bacteria, and bad oral hygiene and periodontal diseases lead to the proliferation of pathogenic bacteria that can cause aspiration pneumonia (Finegold, 1991; Scannapieco, 1999; Japanese Respiratory Society, 2004). The aetiological role of pathogenic flora in aspiration pneumonia is of special concern for neurosurgical patients, because the likelihood of long-term post-operative endotracheal intubation and immobilization are additional risk factors for infectious complications involving the lungs (Erman et al., 2005; Korinek et al., 2005).

Administration of cephalosporins to patients as prophylaxis for high-risk surgical procedures at the time of induction of general anaesthesia is routinely carried out in many institutes. In this study the efficacy of pre-operative cefazolin, cefuroxime and cefamandole in controlling the growth of pathogenic aerobic oral flora in comatose patients undergoing neurosurgical procedures was investigated.

At the time of induction of general anaesthesia for neurosurgical procedure, 10, 11 and 9 comatose patients (Glasgow coma scale $<7$ ) received $1 \mathrm{~g}$ cefazolin, $1.5 \mathrm{~g}$ cefuroxime or $2 \mathrm{~g}$ cefamandole intravenously, respectively. Clinical data are detailed in Table 1. At the completion of the surgical procedure, saliva samples were obtained from above the cuff of the endotracheal tube and residual serum samples from routine blood testing were simultaneously collected by procedures that were approved by the Ethical Committee of the University of Debrecen. The actual antibiotic concentrations in the serum and saliva samples were determined by capillary electrophoresis (CE) as described previously (Andrasi et al., 2007). The bacteria from the saliva were isolated, and the MICs of the cephalosporins against the specific bacteria were evaluated by the standard broth microdilution method according to the guidelines of the Clinical and Laboratory Standards Institute (CLSI, 2005).

Twenty-one bacteria were isolated from the saliva of patients who received $1 \mathrm{~g}$ cefazolin prior to surgery. The MIC values of only 3 bacteria (Acinetobacter baumannii, Citrobacter freundii and Pseudomonas aeruginosa) were higher than the mean drug concentrations in the serum $\left(68.4 \pm 14.4 \mathrm{mg}^{-1}\right)$, but the MIC values of 16 bacteria exceeded the level of cefazolin in the saliva $\left(<0.5 \mathrm{mg} \mathrm{l}^{-1}\right)$. The concentration of antibiotic is given as the mean $\pm S D$.

Twenty bacterial species were isolated from the saliva of patients who received $1.5 \mathrm{~g}$ cefuroxime prophylactically. Two bacteria (C. freundii and P. aeruginosa) had higher MIC values than the mean antibiotic level in the serum $\left(50.3 \pm 18.2 \mathrm{mg} \mathrm{l}^{-1}\right)$. The concentration of cefuroxime in the saliva $\left(<0.5 \mathrm{mg} \mathrm{l}^{-1}\right)$ remained less than the MIC values of 15 bacteria.

Twenty-four bacteria were isolated from the saliva of patients who received $2 \mathrm{~g}$ cefamandole. Four bacteria (A. baumannii,

Table 1. Clinical parameters of patients

\begin{tabular}{|lccc|}
\hline & Cefazolin & Cefuroxime & Cefamandole \\
\hline No. of cases & 10 & 11 & 9 \\
Age (years) & $53.5 \pm 12.3$ & $49.2 \pm 11.5$ & $46.9 \pm 11.5$ \\
Gender (female/male) & $5 / 5$ & $4 / 7$ & $5 / 4$ \\
Body weight (kg) & $72.0 \pm 15.2$ & $71.1 \pm 12.0$ & $69.3 \pm 12.7$ \\
Duration of surgery (min) & $133.5 \pm 19.5$ & $125.5 \pm 23.7$ & $120.4 \pm 24.3$ \\
\hline
\end{tabular}

C. freundii, Enterobacter cloacae and $P$. aeruginosa) had higher MIC values than the mean drug level in the serum $\left(42.4 \pm 9.9 \mathrm{mg} \mathrm{l}^{-1}\right)$. The MIC values of 18 bacteria exceeded the levels of cefamandole in the saliva $\left(<0.5 \mathrm{mg} \mathrm{l}^{-1}\right)$.

Aspiration of oral bacteria is one of the main aetiological factors in developing pneumonia in hospitals. During lengthy surgical procedures, saliva accumulates in the pharynx above the cuff of the tube used for endotracheal anaesthesia. Such secretions are periodically suctioned out, but this procedure cannot always be executed completely. The risk for postoperative aspiration is especially high in comatose patients because of the impaired function of the lower cranial nerves, resulting in impaired pharyngeal and coughing reflexes that also increase the chance of developing pneumonia (Morgan \& Mackay, 1999; Rosenstock et al., 2001).

Since aspiration of pathogenic oral flora increases the incidence of pneumonia, administration of prophylactic antibiotics in high-risk patients is of enormous clinical importance (Kraus et al., 2005). In this study, the efficacy of prophylactic cefazolin, cefuroxime and cefamandole in controlling the growth of oral pathogenic bacteria was evaluated by monitoring the actual concentrations of antibiotics in the serum and saliva. The detected drug levels in the serum proved to have a significant effect against bacteria isolated from the saliva, but the concentrations of every cephalosporin tested were very low in the saliva; cefazolin, cefuroxime and cefamandole did not reach the MIC values of $76.2,75$ and $75 \%$ of the bacteria, respectively, and therefore had only a very moderate effect on the oral flora, and thus the potential prevention of aspiration pneumonia.

Direct monitoring of the effectiveness of antibiotics is not yet included in any clinical practice, although many previous analyses have called attention to very 
different penetration rates of antibiotics in different secretions. These observations are particularly important for patients in whom bacterial diseases can develop by means of a secretion. During recent investigations CE has been applied and shown to be an inexpensive, simple and effective method that requires very small samples, and offers the opportunity for clinicians to evaluate the actual efficacy of antibiotics thus promoting the optimization of individual antibiotic therapies.

In the case of patients at high risk for the aspiration of pathogenic oral flora, e.g. comatose patients, in place of cefazolin, cefuroxime and cefamandole, a prophylactic antibiotic should be administered that is able to reach the MIC values of the majority of pathogenic bacteria in the saliva. Determination of the MIC values of the bacteria isolated from the saliva collected during the surgical procedure can facilitate specific antibiotic therapy in the case of post-operative aspiration pneumonia.

\section{Acknowledgements}

This study was supported by the Hungarian Ministry of Education (OTKA, no. F-049050).

\section{Kinga Bágyi, ${ }^{1}$ Ildikó Márton, ${ }^{1}$ Judit Szabó, ${ }^{2}$ Melinda Andrási, ${ }^{3}$}

\section{Attila Gáspár, ${ }^{3}$ Imre Varga, ${ }^{4}$ László Bognár ${ }^{5}$ and Almos Klekner ${ }^{5}$}

${ }^{1}$ Faculty of Dentistry, Medical and Health Science Centre, University of Debrecen, Debrecen, Hungary

${ }^{2}$ Institute of Medical Microbiology, Medical and Health Science Centre, University of Debrecen, Debrecen, Hungary

${ }^{3}$ Department of Inorganic and Analytical Chemistry, Medical and Health Science

Centre, University of Debrecen,

Debrecen, Hungary

${ }^{4}$ Department of Pulmonology, Medical and Health Science Centre, University of Debrecen, Debrecen, Hungary

${ }^{5}$ Department of Neurosurgery, Medical and Health Science Centre, University of Debrecen, Debrecen, Hungary

Correspondence: Almos Klekner (aklekner@yahoo.com)

Andrasi, M., Gaspar, A. \& Klekner, A. (2007). Analysis of cephalosporins in bronchial secretions by capillary electrophoresis after simple pre-treatment. J Chromatogr B Analyt Technol Biomed Life Sci 846, 355-358.

CLSI (2005). Performance Standards for Antimicrobial Susceptibility Testing, 15th informational supplement, approved standard M7-A6. Wayne, PA: Clinical and Laboratory Standards Institute.
Erman, T., Demirhindi, H., Gocer, A. I., Tuna, M., Ildan, F. \& Boyar, B. (2005). Risk factors for surgical site infections in neurosurgery patients with antibiotic prophylaxis. Surg Neurol 63, 107-112.

Finegold, S. M. (1991). Aspiration pneumonia. Rev Infect Dis 13, S737-S742.

Korinek, A. M., Golmard, J. L., Elcheick, A., Bismuth, R., van Efferenterre, R., Coriat, P. \& Puybasset, L. (2005). Risk factors for neurosurgical site infections after craniotomy: a critical reappraisal of antibiotic prophylaxis on 4,578 patients. Br J Neurosurg 19, 155-162.

Kraus, D. H., Gonen, M., Mener, D., Brown, A. E., Bilsky, M. H. \& Shah, J. P. (2005). A standardised regimen of antibiotics prevents infectious complications in skull base surgery. Laryngoscope 115, 1347-1357.

Morgan, A. S. \& Mackay, L. E. (1999). Causes and complications associated with swallowing disorders in traumatic brain injury. J Head Trauma Rehabil 14, 454-461.

Rosenstock, C., Moller, J. \& Hauberg, A. (2001). Complaints related to respiratory events in anaesthesia and intensive care medicine from 1994 to 1998 in Denmark. Acta Anaesthesiol Scand 45, 53-58.

Scannapieco, F. A. (1999). Role of oral bacteria in respiratory infection. J Periodontol 70, 793-802.

Japanese Respiratory Society (2004). Aspiration pneumonia. The committee for the Japanese Respiratory Society guidelines in management of respiratory infections. Respirology 9, S35-S37. 